\title{
An ultrasonic shake-up
}

\author{
Ultrasonic radiation forces are harnessed to trap and then shake clusters of spheres - mimicking the effect of \\ temperature on cluster formation in granular systems. This assembly process has applications from the nanoscale \\ to the macroscale.
}

\section{Bruce Drinkwater}

in $\mathrm{n}$ the 1980s, the Jet Propulsion Laboratory in the USA developed acoustic levitation systems for holding objects in place in a contactless way in the shuttle's spacelab ${ }^{1}$. You might have thought things floated on-board already, but the shuttle actually experiences lots of small accelerations to keep its position and orientation in orbit just right. In the spacelab experiment, with the gravitational field eliminated, liquid drops were ultrasonically suspended and rotated, their surface tension modelling the effect of self-gravity of much larger bodies of matter. In this way, a small levitated liquid drop became a model of the dynamic behaviour of planets and stars. And from this work, diverse applications of ultrasonic levitation have emerged, such as the formation of microreactors, and containerless materials processing and analysis ${ }^{2-5}$. Now, writing in Nature Physics, Melody Lim and colleagues have added a new dimension to this rich history by modelling the assembly of granular clusters ${ }^{6}$.

Ultrasonic levitation uses the momentum inherent in sound waves to apply forces that can overcome gravity ${ }^{7}$. In a typical ultrasonic levitator, high-frequency sound waves are emitted into a cavity, exciting one of its resonant frequencies and leading to the formation of a standing wave. The resulting ultrasonic pressure field consists of a line of pressure minima (nodes) and maxima (antinodes). Solid or liquid particles in the cavity experience net radiation forces towards the nodes, where they become trapped.

Lim and co-workers detuned their cavity by exciting it at a frequency just off-resonance. In addition to the standing wave, this induced travelling waves that repeatedly perturbed the particles, thereby providing a non-contact particle shaking force. When multiple particles were introduced into the node, each pair experienced interparticle attractive forces due to multiple scattering of the sound between particles. The magnitude of these forces depended on the particle size relative to the wavelength. In this way, Lim and colleagues were able to independently tune the shaking forces and the interparticle forces by controlling the amount of detuning of the
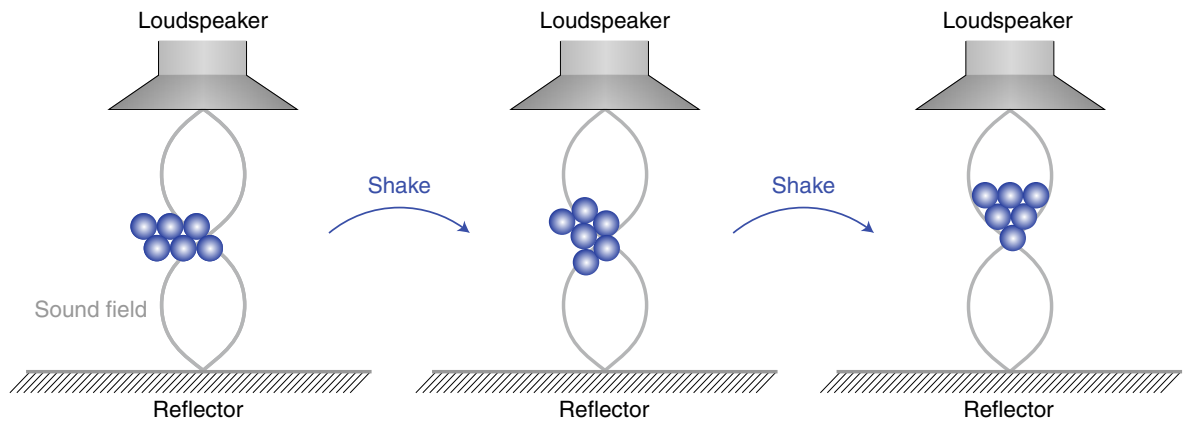

Fig. 1 | Shaking granular clusters with ultrasonic radiation. Using their ultrasonic levitator, Lim and colleagues were able to image the dynamic behaviour of clusters of millimetre-sized spheres. By shaking the system, they induced statistically predictable jumps between different stable cluster patterns.

excitation, its amplitude and the wavelength of the sound. In short, they created an easyto-observe, tunable, macroscale model of a non-equilibrium granular system.

The authors used this ultrasonic levitator and shaker to study the dynamic behaviour of clusters of millimetre-sized spheres (Fig. 1 ). As the effective temperature of five-, six- and seven-particle clusters was changed by more violent shaking, the clusters were seen to jump between different stable patterns along pathways whose probability was predictable. Given the small number of particles in the cluster, most of the clusters were $2 \mathrm{D}$, but at higher temperatures, jumps to $3 \mathrm{D}$ structures started to emerge. In this way, their method opens up new means of understanding the assembly of granular matter, which may have applications in creating materials with finely controlled structures. Their approach can be tuned to represent a wide range of physical systems on any scale, for example microscale colloidal crystals. All that is needed is an ultrasonic levitator and a slow-motion camera.

The other good news is that ultrasonic levitation experiments are not costly to set up. Over the last few years, low-cost ultrasonic levitators and drive electronics have emerged ${ }^{8,9}$. These use an array of low-cost parking sensors as the ultrasonic emitters and circuitry designed for consumer audio applications to provide the excitation. Using these published designs, an ultrasonic levitator system can be built for around US\$100. To observe the particle dynamics seen by Lim and colleagues, a moderate-specification slow-motion camera (>500 frames per second) and suitable image processing software are also needed, but these are now readily available at modest cost. This all means that ultrasonic levitator experiments are accessible to a wide range of researchers - and even to budding scientists who are still in the classroom.

Bruce Drinkwater

Department of Mechanical Engineering, University of Bristol, Bristol, UK.

e-mail:B.Drinkwater@bristol.ac.uk

Published online: 4 March 2019

https://doi.org/10.1038/s41567-019-0463-2

References

1. Wang, T. G., Trinh, E. H., Croonquist, A. P. \& Elleman, D. D. Phys. Rev. Lett. 56, 452-455 (1986).

2. Zang, D. et al. Adv. Colloid Interfac. 243, 77-85 (2017).

3. Owens, C. E., Shields, C. W., Cruz, D. F., Charbonneau, P. \& López, G. P. Soft Matter 12, 717-728 (2016).

4. Seddon, A. M. et al. J. Phys. Chem. Lett. 7, 1341-1345 (2016).

5. Cristiglio, V. et al. Biochim. Biophys. Acta 1861, 3693-3699 (2017).

6. Lim, M. X., Souslov, A., Vitelli, V. \& Jaeger, H. M. Nat. Phys. https://doi.org/10.1038/s41567-019-0440-9 (2019).

7. Andrade, M. A. B., Pérez, N. \& Adamowski, J. C. Braz. J. Phys. 48 190-213 (2018)

8. Marzo, A., Barnes, A. \& Drinkwater, B. W. Rev. Sci. Instrum. 88, 085105 (2017).

9. Marzo, A., Corkett, T. \& Drinkwater, B. W. IEEE Trans. Ultrason. Ferroelectr. Freq. Control 65, 102-111 (2018). 